\title{
RETIFICADOR DE 6KW, FATOR DE POTÊNCIA UNITÁRIO, TRIFÁSICO, COMUTAÇÃO NÃO DISSIPATIVA NA CONVERSÃO CC-CC E CONTROLE SINCRONIZADO EM FREQÜÊNCIA.
}

\author{
Carlos H. G. Treviso ${ }^{1}$, Lúcio R. Barbosa ${ }^{1}$, Adriano A. Pereira ${ }^{2}$, João B. Vieira $\mathrm{Jr}^{2}$ e Luiz C. Freitas ${ }^{2}$ \\ (')E-mails: treviso@uel.br, 1barbosa@uel.br \\ Universidade Estadual de Londrina \\ CEP 86051-990 - Londrina - PR \\ Brasil
$\left(^{2}\right)$ E-mails: batista@ufu.br, freitas@ufu.br, adriano@eletrica.ufu.br \\ Universidade Federal de Uberlândia \\ CEP 38400-902 - Uberlândia - MG - Brasil
}

\begin{abstract}
Resumo - Este artigo apresenta o funcionamento de um retificador trifásico, fator de potência unitário, comutação não dissipativa na conversão CC-CC e controle sincronizado em freqüência, funcionando com 89\% de rendimento global com baixo nível de RFI. A estrutura proposta permite o funcionamento com alta freqüência de chaveamento. A tensão de saída é controlada por "Modulação por Largura de Pulso (PWM)" com uma freqüência constante. O princípio de funcionamento, análise teórica da conversão $\mathrm{CC}$, equações relevantes, técnica de controle para o balanceamento de corrente, resultados experimentais são mostrados neste artigo.
\end{abstract}

Abstract - This paper presents the operation of a three phase rectifier, unity power factor, non dissipative commutation in the DC-DC conversion and control synchronized in frequency, working with $89 \%$ of global efficiency with low level of EMI. The structure proposal allows the operation with high switching frequency. The output voltage controlled by PWM with a constant frequency. The operational principle, theoretical analysis of the DC conversion, relevant equations, control technique for the current balance, experimental results are shown in this paper.

\section{NOMENCLATURA}

Lr Indutor de ressonância.

$\mathrm{Cr}$ Capacitor de ressonância.

Fs Frequiência de chaveamento

Fo Freqüência de ressonância.

ஸo Pulsação angular de ressonância.

$\alpha$ condutância normalizada.

\section{I - INTRODUÇÃO}

No Brasil, poucos setores estão preocupados realmente com RFI. Somente as indústrias nacionais que produzem equipamentos para os exigentes mercados externos e para as empresas de telecomunicações são obrigadas a realizarem testes de RFI em seus equipamentos [1 - 4].

Através de contatos com fabricantes de renome na produção de retificadores, concluiu-se que a construção de um retificador, capaz de fornecer uma corrente de saída de
$100 \mathrm{~A}$ e uma tensão de $60 \mathrm{~V}$ em corrente contínua, além das características expostas no resumo e tomando-se especial cuidado com os ruídos de RFI, com acabamento semiindustrial, seria uma valiosa contribuição técnico-científica para o mercado nacional [5].

No sentido de ilustrar os objetivos deste trabalho, a figura 1 traz o diagrama de blocos do retificador proposto.

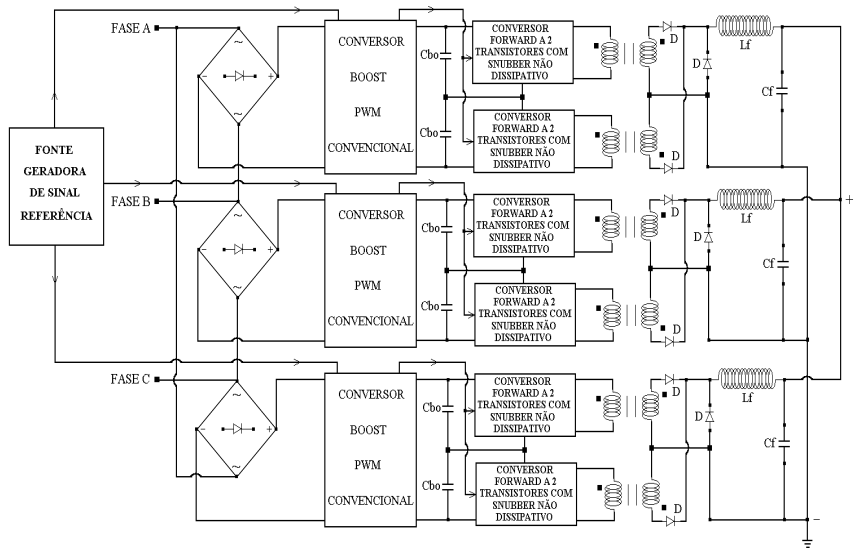

Figura 1 - Diagrama de Blocos do Retificador Trifásico.

Para o estágio CA-CC, utiliza-se o conversor Boost PWM convencional como pré-regulador, muito conhecido na literatura especializada e para a conversão CC-CC, utilizase o conversor Forward a 2 transistores com "snubber" não dissipativo multi-nível.

O casamento das freqüências dos módulos, colocam os ruídos provenientes das derivadas de correntes e/ou tensões, em um espectro bem definido, tornando-se assim, mais fácil a atenuação, constituindo-se em uma importante contribuição [6].

\section{II - CIRCUITO DE GERAÇÃO DE SINAIS.}

Neste circuito são implementados os sinais referências necessários para o casamento das freqüências de todos os conversores do retificador trifásico.

A figura 2 mostra o esquema elétrico do circuito. 


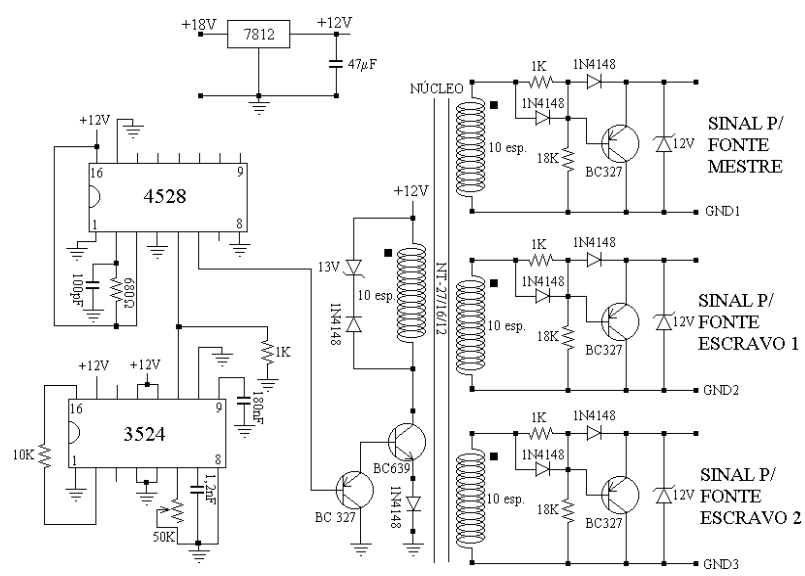

Figura 2 - Esquema Elétrico do Circuito de Geração de Sinais.

Este circuito é colocado em um conjunto $\mathrm{CA}-\mathrm{CC}$ e $\mathrm{CC}-\mathrm{CC}$ e será denominado módulo mestre e os demais conjuntos serão denominados módulos escravos.

\section{III - O CONVERSOR BOOST COMO PRÉ-REGULADOR.}

O conversor Boost, com a devida técnica de controle, possibilita obter-se uma corrente de entrada senoidal e em fase com a tensão de entrada, além de um controle da tensão do barramento CC [7 e 8].

Para a obtenção do exposto acima, utilizou-se o CI3854 para o controle do pré-regulador, cujo diagrama de blocos é mostrado na figura 3 .

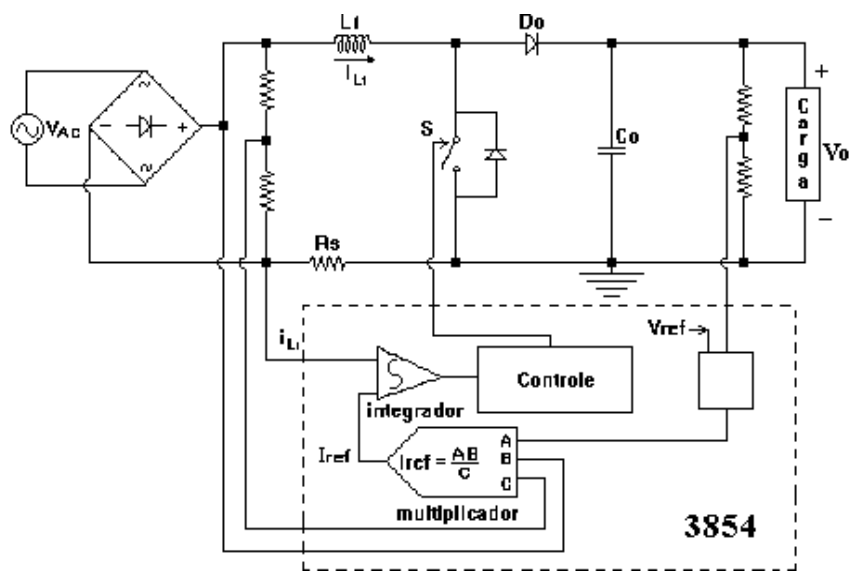

Figura 3 - Diagrama de Blocos do Controle Aplicado ao Conversor Boost.

A técnica utilizada pelo CI3854 é o método de controle da corrente média.

O esquema elétrico completo com todos os componentes dimensionados para a potência de 2200 Watts, tensão de saída de $380 \mathrm{~V}$ e tensão de entrada variando de 185 a 250 VCA, é mostrado na figura 4.

$\mathrm{Na}$ figura 4, o sinal referência proveniente do circuito gerador de sinais faz o transistor BC337 descarregar o capacitor conectado ao pino 14 (3854), casando-se assim a freqüência e o "trimpot" de $12 \mathrm{~K}$ no pino 12 (3854), ajusta a amplitude da triangular. A partir da triangular, faz-se o isolamento do sinal que será utilizado casar a freqüência do circuito de controle do conversor CC-CC.

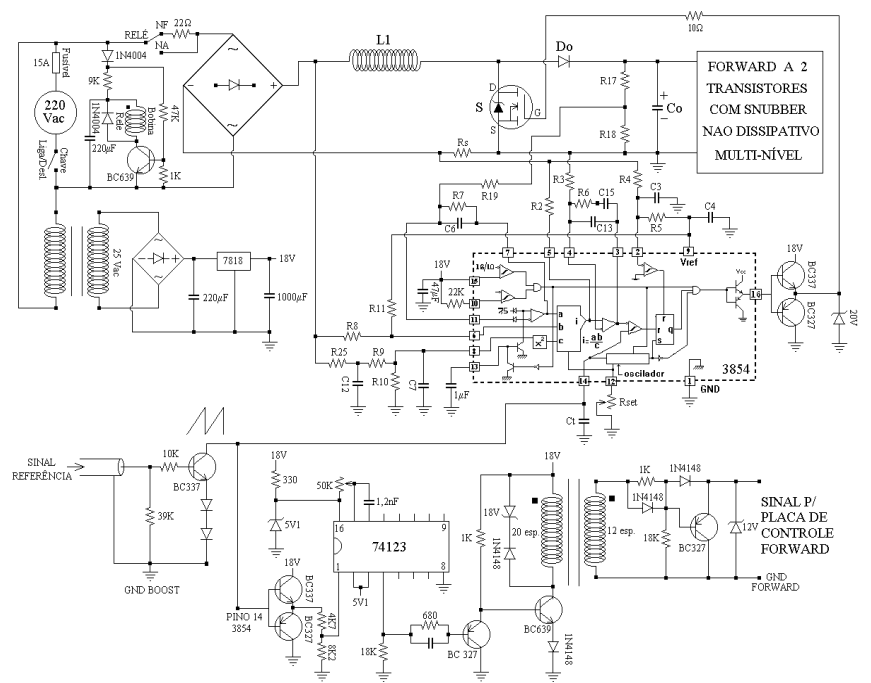

Figura 4 - Esquema Elétrico Completo do Pré-Regulador.

O circuito existente antes da ponte retificadora, faz a limitação da corrente de "inrush", cujo tempo para "curtocircuitar" a resistência de $22 \Omega$ (definido pelo resistor de $9 \mathrm{~K}$ e o capacitor de $220 \mu \mathrm{F}$ ) é 2 s aproximadamente.

\section{IV - A CONVERSÃO CC-CC}

Para a conversão CC-CC, é utilizado o conversor Forward a 2 transistores com "snubber" não dissipativo multi-nível, que consiste em dois conversores Forward a 2 transistores com "snubber" não dissipativo no lado primário e os secundários acoplados. A figura 5 mostra 0 circuito simplificado.

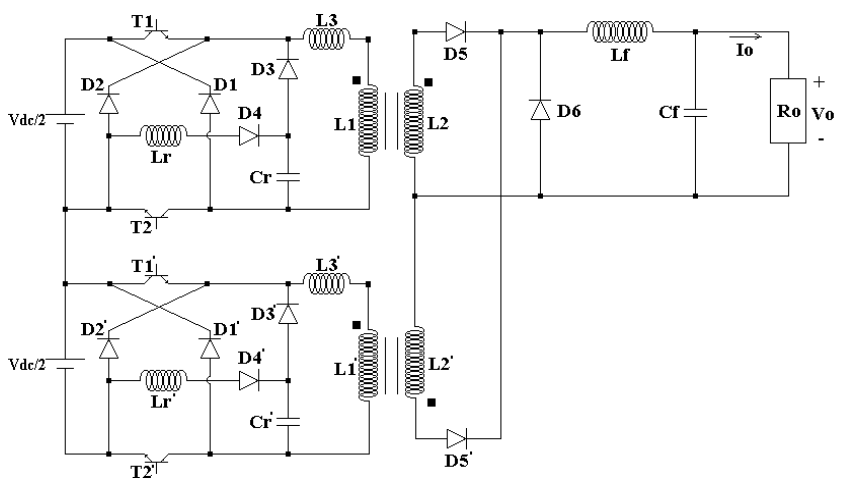

Figura 5 - Circuito Elétrico Simplificado Para a Conversão CC-CC.

As principais vantagens da topologia da figura 5 são: as reduções das tensão nas chaves em $50 \%$ da tensão do barramento (saída do conversor Boost) e o indutor de saída é projetado para o dobro da freqüência de chaveamento de cada conversor Forward. O conversor Boost funciona numa freqüência de $100 \mathrm{KHz}$, enquanto que cada conversor do lado primário funciona a $50 \mathrm{KHz}$, porém defasados de 180 graus.

Para ilustrar o funcionamento da comutação não dissipativa do conversor Forward a 2 transistores, será feita a análise da estrutura da figura 6. 


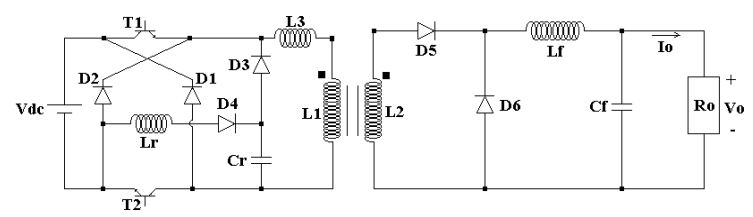

Figura 6 - Circuito simplificado do conversor Forward a 2 Transistor com Snubber não Dissipativo.

Os elementos do "snubber" não dissipativo da figura 6 são: Lr, D3, D4, Cr e L3.

As vantagens desta topologia são: reduzido número de componentes e ausência de um transistor auxiliar para obter a ressonância. Para um projeto bem dimensionado, pode-se obter as mesmas características do conversor Forward a 2 transistores convencional do ponto de vista do controle [9].

A topologia da figura 6 foi analisada de acordo com as seguintes considerações:

- Todos os semicondutores são ideais;

- A tensão de entrada é constante;

- A indutância magnetizante é muito grande;

- Não existe indutância de dispersão;

- A Corrente de saída é constante;

$-\mathrm{N} 1=\mathrm{N} 2$.

Este conversor possui 6 etapas de funcionamento para um ciclo de chaveamento.

PRIMEIRA ETAPA, $\left(\mathbf{t}_{\mathbf{0}}, \mathbf{t}_{\mathbf{1}}\right)$ - Etapa linear de corrente no indutor série L3. Inicia-se quando os transistores T1 e T2 entram em condução simultaneamente com correntes nulas (ZCS), devido a indutância L3. Neste estágio começa a ressonância entre $\mathrm{Lr}$ e $\mathrm{Cr}$, com a tensão no capacitor $\mathrm{Cr}$ variando de $-\mathrm{Vdc}$ até um valor $\mathrm{VCr} 1$, enquanto a corrente no indutor Lr varia de zero a um valor iLr1, terminando esta etapa quando ocorre o bloqueio do diodo de roda livre D6.

SEGUNDA ETAPA, $\left(\mathbf{t}_{\mathbf{1}}, \mathbf{t}_{\mathbf{2}}\right)$ - Este estágio é a etapa PWM. Continua ocorrendo a ressonância entre $\mathrm{Lr}$ e $\mathrm{Cr}$, com a tensão em $\mathrm{Cr}$ variando de $\mathrm{VCr} 1 \mathrm{a}+\mathrm{Vdc}$ e a corrente iLr1 atinge um máximo e decresce até zero, terminando esta etapa quando os transistores T1 e T2 entram em corte.

TERCEIRA ETAPA, $\left(\mathbf{t}_{\mathbf{2}}, \mathbf{t}_{\mathbf{3}}\right)$ - Nesta etapa ocorre o descarregamento linear do capacitor de ressonância. Inicia-se com a abertura dos transistores T1 e T2 sob tensões nulas (ZVS) devido ao capacitor de ressonância estar carregado com a tensão de entrada Vdc. A corrente de saída constante impõe uma descarga linear do capacitor $\mathrm{Cr}$, chegando a tensão nula quando termina esta etapa.

QUARTA ETAPA, $\left(\mathbf{t}_{\mathbf{3}}, \mathbf{t}_{\mathbf{4}}\right)$ - Nesta etapa ocorre a ressonância entre o capacitor $\mathrm{Cr}$ e a indutância magnetizante do transformador. Este estágio inicia-se quando a tensão no capacitor torna-se nula, polarizando diretamente o diodo de roda livre D6, devido a corrente de saída Io. A energia do transformador é transferida para o capacitor $\mathrm{Cr}$, invertendo sua polaridade, terminando esta etapa quando a tensão em $\mathrm{Cr}$ atingir -Vdc.

QUINTA ETAPA, ((t) $\left.\mathbf{t}_{\mathbf{5}}\right)$ - Nesta etapa tem-se a desmagnetização do transformador. $\mathrm{O}$ início ocorre quando a tensão do capacitor atinge -Vdc, polarizando os diodos D1 e D2. A corrente magnetizante circula através dos diodos, devolvendo energia para a fonte de entrada, terminando este estágio quando a corrente magnetizante torna-se nula.

SEXTA ETAPA, $\left(\mathbf{t}_{\mathbf{5}}, \mathbf{t}_{\mathbf{6}}\right)$ - Nesta etapa ocorre o grampeamento da tensão do capacitor $\mathrm{Cr}$ em -Vdc. Inicia-se quando a corrente magnetizante torna-se nula, permanecendo neste estado até ocorrer os disparos dos transistores. Quando termina este estágio, fecha-se o ciclo de chaveamento.

A figura 7 mostra os circuitos equivalentes para cada etapa de funcionamento.
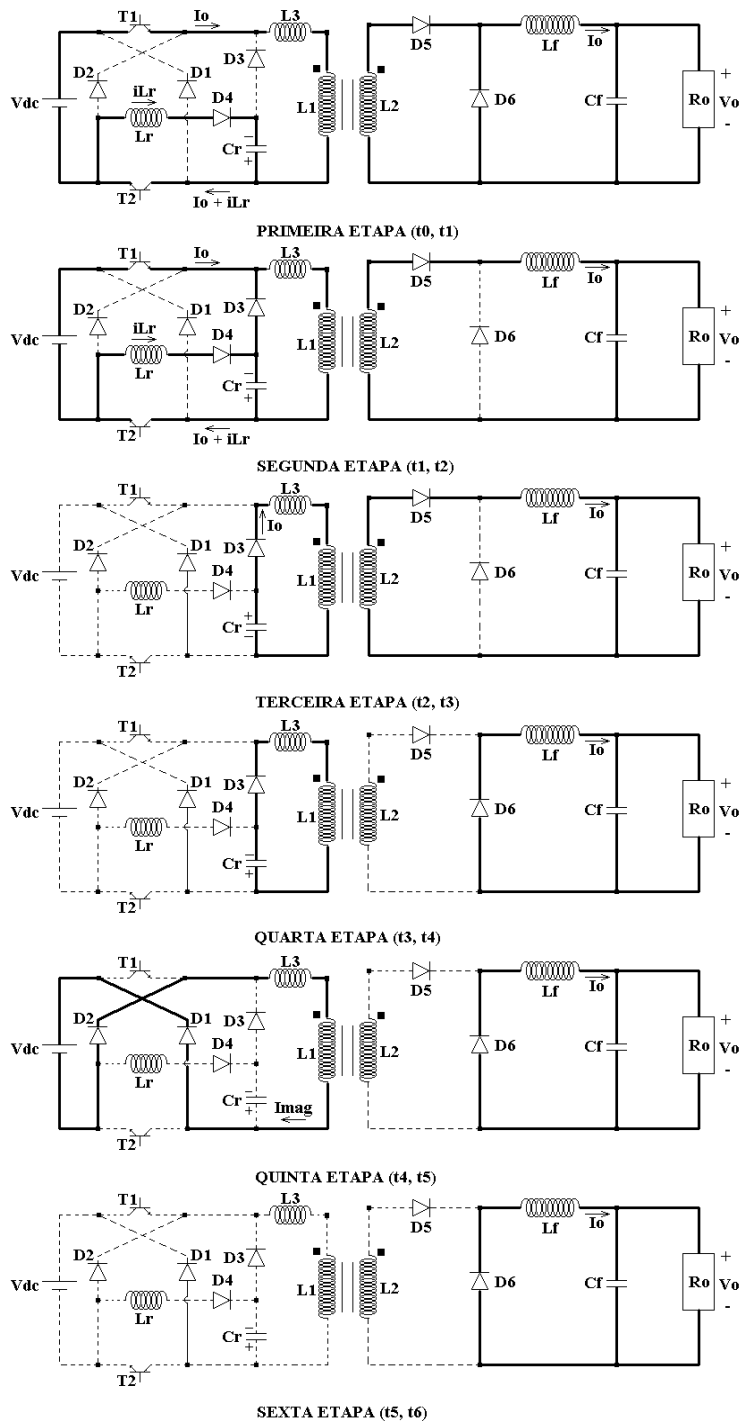

Figura 7 - Circuitos Equivalentes para Cada Etapa de Funcionamento Para Um Ciclo de Chaveamento.

As etapas de funcionamento deram origem às formas de onda mostrada na figura 8 . 


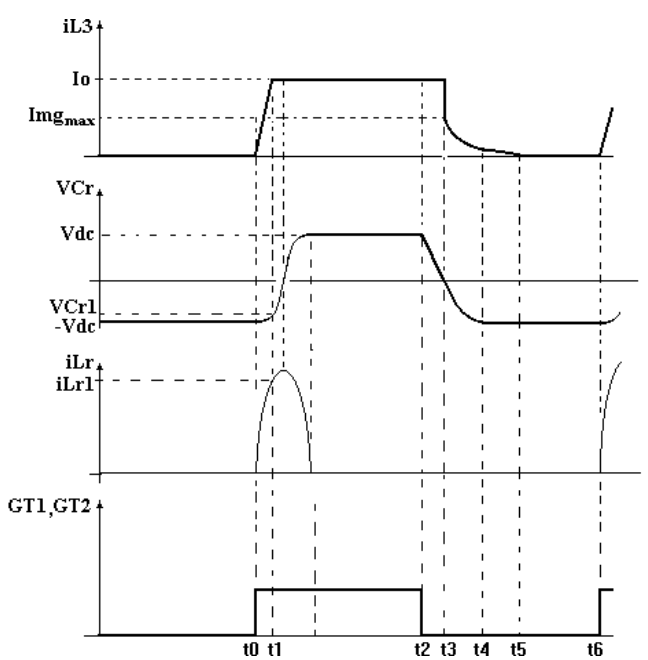

Figura 8 - Principais Formas de Onda Para os Circuitos Equivalentes.

A tensão de saída Vo, pode ser obtida através da análise dos estágios de funcionamento com as considerações expostas anteriormente para análise.

Partindo das considerações acima, chega-se na equação 1:

$$
\begin{aligned}
\frac{V O}{V d c} & =d-F S \cdot \frac{\alpha}{\omega o}+\frac{F S}{2 \cdot \omega o \cdot \alpha} \\
\omega O & =2 \pi F O=\frac{1}{\sqrt{L_{R} C_{R}}} \\
\alpha & =\frac{\left(I_{0}\right)}{V d c} \sqrt{\frac{L_{R}}{C_{R}}}
\end{aligned}
$$

Onde:

$F S=$ freqüência de chaveamento;

$F O=$ freqüência de ressonância;

$d=$ razão cíclica.

$I o=$ Corrente de carga.

A figura 9 mostra a faixa de conversão g para as equações acima.

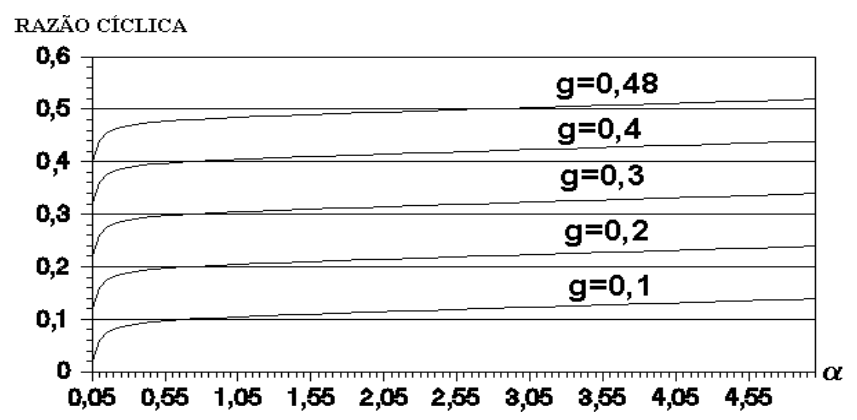

Figura 9 - Faixa de conversão para o conversor.

De acordo com a figura 9, este conversor apresenta uma diminuição do ganho estático em relação a razão cíclica para $\alpha$ 's maiores. Isto acontece porque para cargas maiores, os tempos de descargas do capacitor de ressonância tornam-se menores, resultando em uma tensão média positiva menor no primário do transformador.

\section{V - A ESTRATÉGIA DE CONTROLE PARA A CONVERSÃO CC-CC.}

Para realizar do controle da conversão CC-CC de cada módulo, será utilizado o CI3525.

O sincronismo da freqüência de chaveamento do conversor CC-CC com a freqüência do conversor Boost é realizado através do circuito elétrico mostrado na figura 10 .

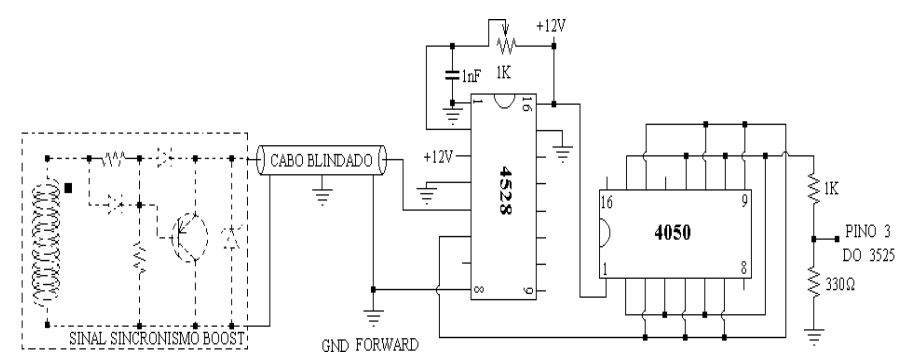

Figura 10 - Circuito Para Sincronizar Com Freqüência do Conversor Boost.

O pino 3 do CI3525 é a entrada para se fazer o sincronismo. O sinal proveniente do conversor Boost (figura 4) possui isolação galvânica e entra no monoestável, produzindo pulsos de $2,8 \mathrm{~V}$ e largura de $0,5 \mu \mathrm{s}$, realizando assim o casamento de freqüência.

A figura 11 mostra o diagrama de blocos para a realização do controle do módulo mestre.

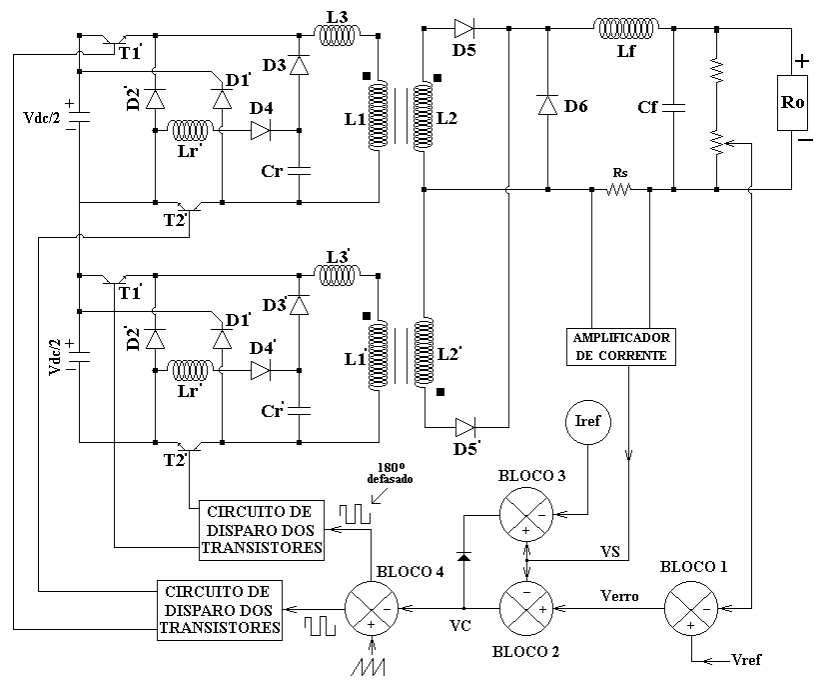

Figura 11 - O diagrama de blocos da estratégia de controle.

A tensão de saída é mostrada e comparada com uma tensão referência produzindo um sinal Verro (bloco 1). O amplificador de corrente produz um sinal de tensão Vs proporcional à corrente de saída. O sinal VS é comparado com os sinais Verro (bloco 2) e Iref (bloco 3). O sinal Vc pode ser gerado a partir dos blocos 2 ou 3 dependendo do valor de Iref e comparado com uma dente de serra (bloco 4), produzindo os pulsos para as chaves. O bloco circuito de disparo dos transistores faz a isolação galvânica dos pulsos.

A figura 12 mostra o diagrama de blocos para a fonte trifásica. 


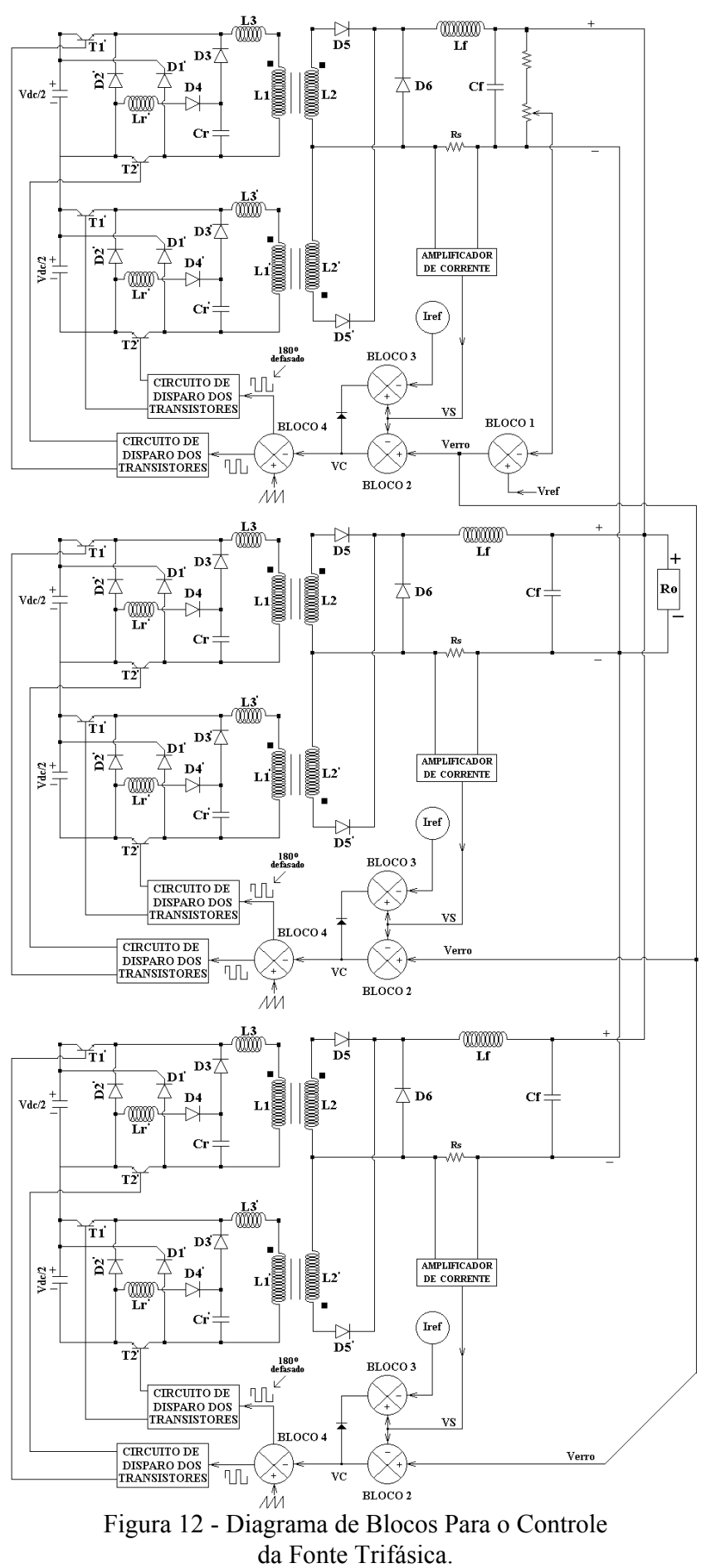

Os terminais positivo e negativo de cada módulo estão conectados em paralelo, consequentemente, ao mesmo potencial. Considerando que os valores dos sensores de corrente são iguais, e implementando a realimentação da tensão de saída no módulo mestre, tem-se a tensão Verro e esta irá para os módulos escravos. Em cada módulo, o circuito responsável para a obtenção do sinal de tensão Vs possui o mesmo ganho, e irá acompanhar o sinal de Verro. O mesmo princípio é válido para Iref, ou seja, Iref é comum a todos os módulos.

\section{VI - EXEMPLO DE PROJETO}

O projeto deve ser feito com os valores adequados de $\alpha$ (maior que 0,3 ) para que o conversor funcione na região PWM como mostrado na figura 9. Uma outra condição a ser satisfeita é que a freqüência de ressonância seja pelo menos dez vezes maior que a freqüência de chaveamento. Desta forma pode-se calcular os valores dos elementos ressonantes que compõem a estrutura. As equações básicas para o cálculo do indutor e do capacitor de ressonância são aquelas dadas de (2) a (3). Os valores dos elementos ressonantes devem satisfazer os valores de $\alpha$ para que o conversor esteja na região de funcionamento PWM.

Dados do projeto:

Tensão de entrada $(\mathrm{Vdc})=300 \mathrm{~V}$;

Corrente de entrada (Io) $=20 \mathrm{~A}$;

Freqüência de Chaveamento $=100 \mathrm{kHz}$;

$\mathrm{Vo}=60 \mathrm{~V} ;$ Iout $=100 \mathrm{~A} ; \mathrm{Po}=6000 \mathrm{~W}$ e $\alpha=1$.

$\frac{f_{S}}{f_{o}}=0,1 \Rightarrow f_{o}=1 \mathrm{MHz}$

$1 * 10^{6}=\frac{1}{2 \pi \sqrt{L r \cdot C r}} \Rightarrow C r=\frac{1}{4 * 10^{12} \pi^{2} \cdot \operatorname{Lr}}$

$$
\begin{aligned}
& \alpha=\frac{20}{300} \cdot \sqrt{\frac{L r}{C r}} \Rightarrow 1^{2}=\frac{400}{90000} \cdot L r^{2} \cdot 39,5 * 10^{12} \\
& L r=2,39 \mu H \Rightarrow C r \cong 10,5 \eta F
\end{aligned}
$$

Pelo fato do valor de $\mathrm{Cr}$ não ser um valor comercial, adota-se um capacitor de $10 \mathrm{nF}$. Com isto, tem-se $\alpha=1,03$, e o valor de $f o=1,03 \mathrm{MHz}$.

\section{VII - TÉCNICAS DE SUPRESSÃO DE RFI.}

Os testes RFI possuem custos elevadíssimos, pois são realizados em laboratórios específicos. Sendo assim, antes de realizar aos testes, deve-se ter certeza de que o equipamento desenvolvido esteja com níveis aceitáveis, para não ocorrer um dispêndio financeiro desnecessário. Com isso, adota-se métodos práticos e eficientes para verificar se o equipamento sob teste tem baixo RFI. Para isto, basta ligar o equipamento próximo a equipamentos que funcionam com freqüências diversas, tais como televisores, rádios AM e FM. A verificação será visual e/ou auditiva. No caso da TV, aparecerão faixas, indicando que o equipamento precisa de filtros. Quanto ao Rádio, acorrerão "assovios" [5, 6 e 10]. Foram adotadas diversas técnicas para conseguir-se atenuações dos ruídos conduzidos e radiados, tais como: cuidado especial com o "lay-out", separação entre primário e secundário, utilização dos capacitores tipo $\mathrm{X}$ e $\mathrm{Y}$ para supressão dos ruídos modo comum e diferencial, blindagens nos transformadores e indutores, desacoplamento dos transformadores e indutores, blindagem do módulo, filtro de entrada blindado e "snubber" dissipativo em todos os semicondutores de potência.

A figura 13 mostra o circuito elétrico projetado para um módulo, adotando todos a técnicas para a reduções dos ruídos RFI. 

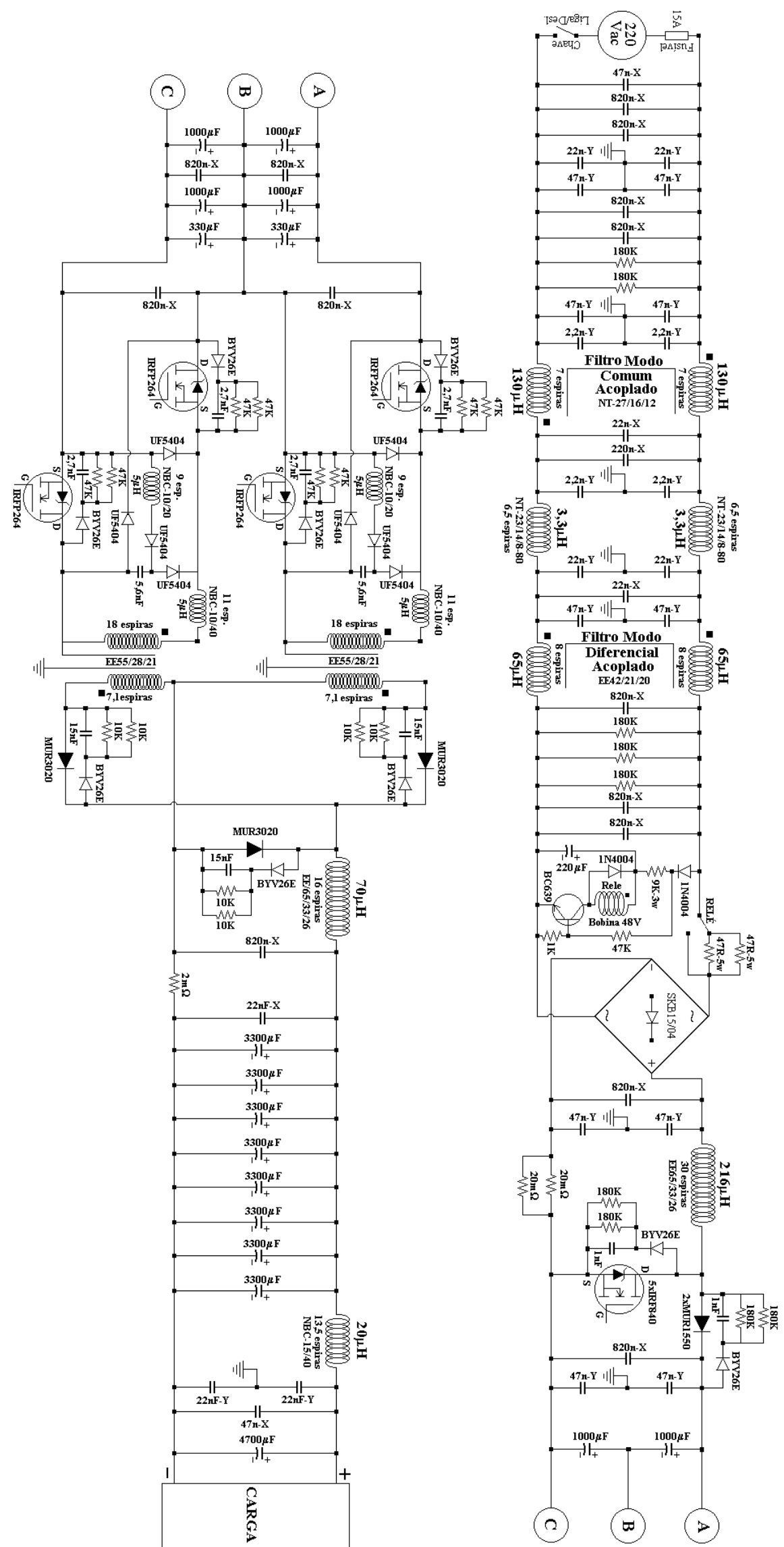

Figura 13 - Esquema Elétrico da Potência de Cada Módulo. 
Ao ligar a fonte trifásica próximo a TV, verificou-se faixas transversais praticamente imperceptíveis e quanto ao rádio, não observou-se qualquer alteração no som, indicando que a fonte possui baixo RFI.

\section{VIII - RESULTADOS EXPERIMENTAIS.}

Os resultados experimentais são obtidos do módulo mestre, pois a técnica de controle empregada, faz com que os demais módulos tenham o mesmo comportamento e a mesma resposta dinâmica da fonte trifásica. Além disso, o laboratório possui somente um Wattímetro “..TRUE RMS...", o que reforçou a decisão de obter-se os resultados somente do módulo mestre (medidas mais confiáveis).

$\mathrm{Na}$ figura 14 tem-se a forma de onda da tensão e corrente de entrada para a potência de 2000 Watts de saída.

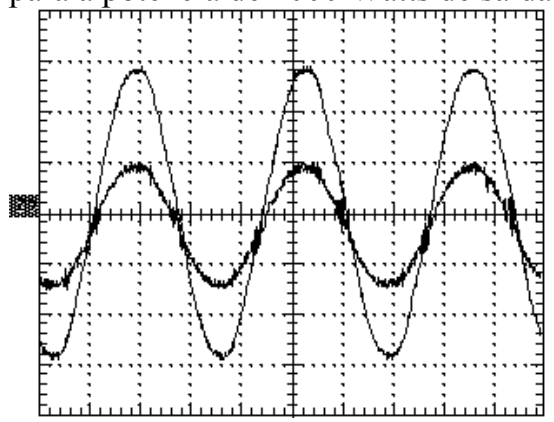

Figura 14 - Formas de Onda da Tensão e Corrente de Entrada Para Potência de 2000W de Saída.

A figura 15 mostra a curva do fator de potência para potências variando de $300 \mathrm{~W}$ a $2000 \mathrm{~W}$ de saída.

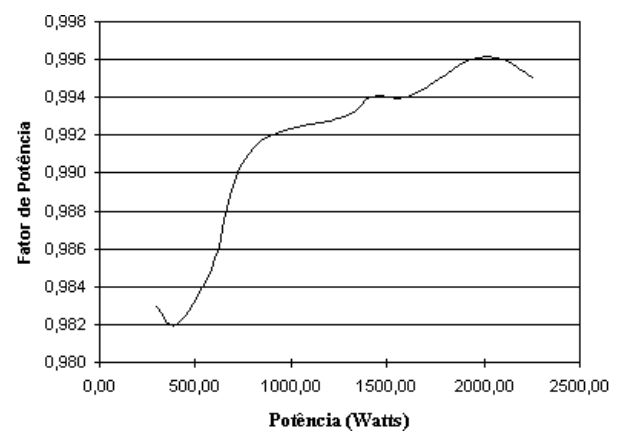

Figura 15 - Curva do Fator de Potência.

A figura 16 traz a forma de onda da tensão e corrente no mosfet que faz parte do circuito ressonante do conversor Forward a 2 transistores com "snubber" não dissipativo.

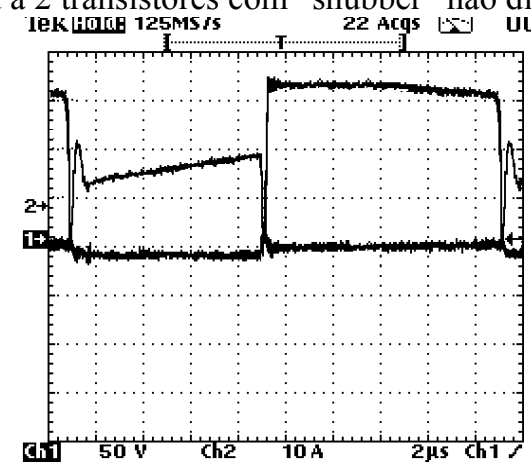

ov

Figura 16 - Forma de Onda da Tensão e Corrente no Mosfet do Circuito Ressonante.

A figura 17 traz o rendimento de cada módulo.

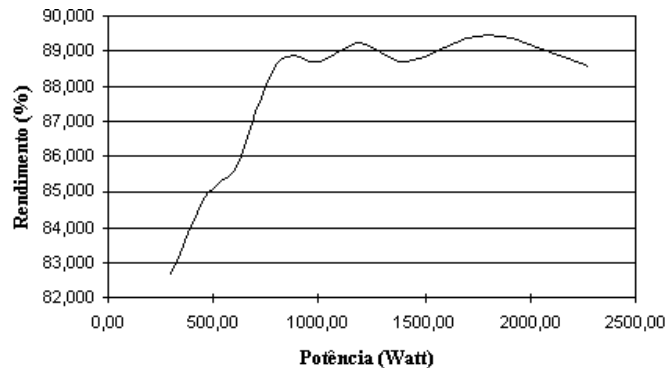

Figura 17 - Rendimento Global do Módulo Monofásico.

A figura 18 expõe a resposta dinâmica para a fonte trifásica, com variação de carga de $50 \%$ a $100 \%$ e freqüência de $1 \mathrm{KHz}$.

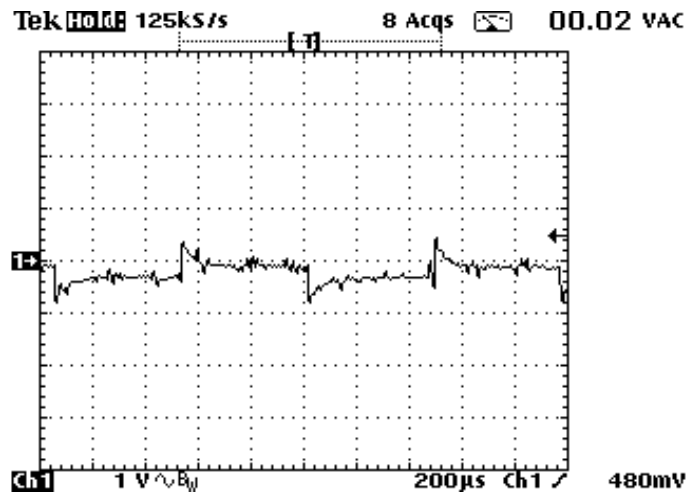

Figura 18 - Resposta Dinâmica da Fonte Trifásica Com Carga Variando de $3000 \mathrm{~W}$ a $6000 \mathrm{~W}$.

Observa-se que o controle possui ótimo desempenho, pois não se verificou oscilações no momento da variação de carga.

A figura 19 mostra a fotografia da fonte trifásica.

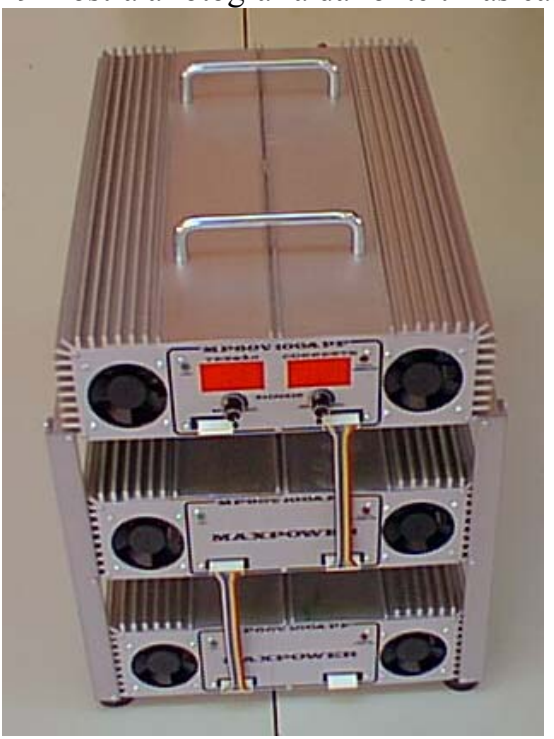

Figura 19 - Fotografia da Fonte Trifásica.

\section{IX - CONCLUSÃO}

Este artigo teve como objetivo, apresentar um retificador trifásico com fator de potência unitário, baixa distorção harmônica de corrente, reduzidos níveis de ruídos radiado e conduzido, alto rendimento, tensão de saída com isolação galvânica e potência de $6 \mathrm{KW}$ de saída $(60 \mathrm{~V}, 100 \mathrm{~A})$. 
Isto tornou-se possível com a utilização do conversor Boost PWM convencional como pré-regulador e para a conversão CC-CC, dois conversores Forward a 2 transistores com "snubber" não dissipativo acoplados, ou seja, multinível.

O custo de material da fonte foi estimado em US\$ 1.326,13 dólares, cotados em São Paulo. Levando em consideração o lucro da empresas que varia de 40 a $50 \%$, o custo de material do equipamento ficaria bastante reduzido se for produzido em série e adquirindo-se os componentes diretamente dos fabricantes.

Os resultados experimentais comprovam a eficiência da fonte retificadora trifásica, com rendimento global de $89 \%$ e fator de potência de 0,996 para a potência nominal.

A estrutura da fonte colocados em módulos com divisões de potências, possibilita especificar uma fonte para qualquer faixa de potência, não ficando limitada somente na estrutura trifásica.

Esta fonte pode ser utilizada em telecomunicações, laboratórios experimentais e até para soldagem de peças metálicas.

\section{REFERÊNCIAS BIBLIOGRÁFICAS.}

[1] D. R. J WHITE; EMI Control Methodology And Procedures - EMC Design Syntesis, Don White Consultants Inc, First Edition 1981.

[2] J. S HILL; D. R. J WHITE; EMC HandbookEletromagnetic Interference And Compatibility, Don White Consultants Inc, Second Edition, 1981,.

[3] SPRAGUE, Electric Company Staff Interference Control Field Service Department; "Interference Control Techniques”, Sprague Technical Paper No. 62-1, Sprague Eletric Company.

[4] COMITÊ BRASILEIRO DE ELETRICIDADE; "III Seminário Brasileiro de Radiointerferência e Compatibilidade Eletromagnética", Anais 1988.

[5] C. H. G TREVISO; Retificador de 6KW, Fator de Potência Unitário, trifásico, Comutação não Dissipativa na Conversão CC/CC e Controle Sincronizado em Freqüencia, Tese de Doutorado, Uberlândia - MG, Março/99.

[6] D. F da CRUZ; Contribuição ao Estudo dos Fenômenos de Radio-Interferência Provocados Por Fontes Chaveadas: Análise, Propostas de Solução e Resultados Experimentais, Dissertação de Mestrado, Uberlândia, 1996.

[7] PROFESSIONAL EDUCATION SEMINARS WORKBOOK; Power factor Correction Circuits Topologies \& Control - Section 4, APEC'93.

[8] C. S. e SILVA; Power Factor Correction With The UC3854, UNITRODE, pp. 9/287-9/296.

[9] C.H.G. TREVISO; A. A. PEREIRA; L. C. FREITAS; V. J. FARIAS; J. B. VIEIRA Jr; A 1,5 KW Two Transistors Forward Converter Using Non-Dissipative Snubber, IEEE PESC'98, pp. 696-700, Maio/98, Fukuoka, Japão.
[10] ERICSSON STAFF; Pratical Methods For Electromagnetic Interference Control, Ericsson.

[11] L.C. de FREITAS; P.R.C. GOMES; A High-Power High-Frequency ZVS-ZCS-PWM Buck Converter Using a Feedback Resonant Circuit, IEEE PESC'93 Record, pp. 330-336.

[12] L.F.P. de MELLO; Projetos de Fontes Chaveadas, Érica, $3^{\mathrm{a}}$ Edição.

[13] C.H.G. TREVISO; A.V. da COSTA; L.C. de FREITAS; A New ZVS-ZCS-PWM Boost Converter With Unit Power Factor Operation, IEEE APEC'94, pp. 404-410.

\section{DADOS BIOGRÁFICOS}

Carlos Henrique Goncalves Treviso, nascido em 05/05/1968 em Pontal-SP, é engenheiro eletricista (1992), mestre (1994) e doutor em Engenharia Elétrica (1999) pela Universidade Federal de Uberlândia. Atualmente é professor adjunto da Universidade Estadual de Londrina. Suas áreas de interesse são: eletrônica de potência, sistemas de controle eletrônicos e circuitos para correção do fator de potência.

Lúcio dos Reis Barbosa, nascido em 19/01/1969 em Patos de Minas-MG, é engenheiro eletricista (1993), mestre (1996) e doutor em Engenharia Elétrica (2000) pela Universidade Federal de Uberlândia. Atualmente é professor adjunto da Universidade Estadual de Londrina. Suas áreas de interesse são: eletrônica de potência, conversão de potência em alta freqüência, correção do fator de potência e novas topologias de conversores.

Adriano Alves Pereira, nascido em 06/06/1964 em Pratápolis-MG, é engenheiro eletricista (1987), mestre (1995) e doutor em Engenharia Elétrica (1998) pela Universidade Federal de Uberlândia. Atualmente é professor adjunto da Universidade Federal de Uberlândia. Suas áreas de interesse são: eletrônica de potência, qualidade da energia elétrica, sistemas de controle eletrônicos e microeletrônica.

João Batista Vieira Júnior, nascido em 23/04/1955 em Panamá-GO, é engenheiro eletricista (1980) pela Universidade Federal de Uberlândia, mestre (1984) e doutor em Engenharia Elétrica (1991) pela Universidade Federal de Santa Catarina. Atualmente é professor titular da Universidade Federal de Uberlândia. Suas áreas de interesse são: conversão de potência em alta freqüência, modelamento e controle de conversores, circuitos para correção de fator de potência e novas topologias de conversores.

Luiz Carlos de Freitas, nascido em 01/04/1952 em Monte Alegre-MG, é engenheiro eletricista (1975) pela Universidade Federal de Uberlândia, mestre (1985) e doutor em Engenharia Elétrica (1992) pela Universidade Federal de Santa Catarina. Atualmente é professor titular da Universidade Federal de Uberlândia. Suas áreas de interesse são: conversão de potência em alta freqüência, modelamento e controle de conversores, circuitos para correção de fator de potência e novas topologias de conversores. 